This is the pre-peer reviewed version of the following article: Sexual Size Dimorphism and Growth Plasticity in Snakes: an Experiment on the Western Diamond-Backed Rattlesnake (Crotalus atrox) by Emily N. Taylor and Dale F. Denardo published in Journal Of Experimental Zoology 303A(7) Copyright (C) 2005 Wiley-Blackwell, which has been published in final form at http://dx.doi.org/10.1002/jez.a.189

\title{
Sexual Size Dimorphism and Growth Plasticity in Snakes: an Experiment on the Western Diamond-Backed Rattlesnake (Crotalus atrox)

\author{
Emily N. Taylor and Dale F. Denardo
}

\begin{abstract}
We conducted an experiment to examine the effects of sex and food intake on growth, mass gain, and attainment of sexual maturity in Western Diamond-backed Rattlesnakes (Crotalus atrox). We also measured testosterone levels to determine whether testosterone might be involved in the male-biased sexual size dimorphism observed in this species. We collected neonate rattlesnakes and raised them in the laboratory for 2 years on either a high-intake diet (fed one mouse per week) or a low-intake diet (fed one mouse every 3 weeks). High-intake snakes grew and gained mass more rapidly than low-intake snakes, but males did not grow or gain mass more rapidly than females in either treatment group. High-intake snakes attained reproductive maturity earlier than low-intake snakes, indicating that size, not age, is the critical determinant of reproductive maturity. Males had higher levels of testosterone than females but did not grow more quickly, suggesting that testosterone may not affect growth in this species and may therefore not be the proximate determinant of sexual size dimorphism.
\end{abstract}

Darwin (1871) recognized that sexual size dimorphism (SSD) was prevalent among animal species and varied in both direction and magnitude. Mammals and birds tend most often to show male-biased SSD where males are larger than females (Andersson, '94). Reptiles are variable but typically show female-biased SSD where females are larger than males (Fitch, '81). Numerous ultimate explanations of SSD have been proposed, but Darwin's original hypotheses are still the most widely discussed: in species where females are larger, size may provide a fecundity advantage, whereas in species where males are larger, size may lend an intrasexual competition advantage (Darwin, 1871). A recent idea emerging from movements to integrate the fields of physiology, ecology, and evolutionary biology is that SSD and other life history characteristics cannot be fully understood without elucidation of the physiological mechanisms responsible for them (e.g., the "physiology/life-history nexus", Ricklefs andWikelski, 2002). Specifically, to understand howselection operates upon a trait such as body size, we must understand how body size is determined on a proximate level in an organism's life (Duvalland Beaupre, '98).

In ectotherms, body size is strongly dependent on resource availability. Numerous studies have experimentally confirmed that snakes exhibit resource-dependent growth; that is, snakes that consume more food grow more quickly and/or gain mass more quickly than snakes that consume less food (Forsman and Lindell, '96; Scudder-Davis and Burghardt, '96; Bonnet et al., 2001). Reproduction may be similarly regulated by resource availability: snakes that consume more food may reproduce earlier or more often than snakes that face lower resource availability (Ford and Seigel, '94; Lourdais et al., 2002). The plasticity of growth in snakes may be an adaptation to variable environments, in which the ability to speed or slow growth in response to fluctuating resource availability may be favored by natural selection (Partridge and Harvey, '88; Forsman and Lindell,'96). However, the sexes may face different selection pressures that favor different growth patterns. For example, in most species of snakes, females are larger than males, presumably because large body 
size often increases fecundity in females (Ford and Seigel, '89; Madsen and Shine, '94). It may therefore be favorable for females to grow faster and/or for longer time periods than males. Indeed, females grow faster than males fed the same diet in several taxa that show female-biased SSD, including Boa (D. Hardy Sr., personal communication) and Nerodia (Scudder-Davis and Burghardt, '96). However, to our knowledge, no one has examined whether males grow faster than females fed the same diet in species of snakes that show male-biased SSD, and few hypotheses exist regarding what physiological factors may be responsible for sexually dimorphic growth in these species. Beaupre (2002) hypothesized that the high energetic costs of reproduction in female rattlesnakes reduce the amount of energy that can be allocated to growth, resulting in smaller size relative to males. However, it is unknown whether this is the sole factor leading to SSD in rattlesnakes, or whether genetic limitations or otherphysiological factors such as sex hormones contribute to SSD.

The steroid hormone testosterone (T) is responsible for the large body size of males in many vertebrate taxa (reviewed in Bardin and Catterall, '81). It is an anabolic hormone with a variety of growth-promoting effects (Bardin and Catterall,'81; Staub and De Beer, '97). However, the growthpromoting effects of $T$ are not consistent across taxa. In the mammalian literature, $T$ promotes growth in those species in which males are the larger sex (e.g., the rat, Slob and Van Derr Werff Ten Bosch, '75) and inhibits growth in thosespecies in which females are larger (e.g., thegolden hamster, Swanson, '67). Like mammals, both male- and female-biased SSD are observed in snakes (Shine, '78; Andrews, '82). In garter snakes (genus Thamnophis), which exhibit female-biased SSD (Fitch, '81), T inhibits growth and thus leads to a smaller size of males than of females (Crews et al., '85; Lerner and Mason, 2001). In snakes with male-biased SSD, it is possible that T stimulates growth in males; however, this possibility has never been tested.

In this experiment, we examined the effects of diet and sex on growth in the Western Diamond-backed Rattlesnake (Crotalus atrox), a snake with male-biased SSD (Klauber, '72). C. atrox males and females appear to be born the same size and grow at the same rate in the field, but develop SSD at some later point, possibly after the time of sexual maturity (Beaupre et al., '98). Figure 1 is a schematic of such a growth trajectory, with a divergence in growth between males and females occurring after attainment of sexual maturity. Historically, scientists have speculated that males grow larger than females as a result of sexual selection (Shine, '78), since larger male pit vipers tend to win fights for females (e.g., Schuett, '97); however, the physiological mechanism responsiblefor the divergence in growth is unknown.

We raised juvenile $C$. atrox in the laboratory on high- and low-intake diets to test several hypotheses. First, we hypothesized that rattlesnakes exhibit resource-dependent growth, and that males and females differ in growth. We predicted that snakes consuming more food would grow faster than snakes consuming less food, and males would grow faster than females. Such a sex difference could result from several factors, including, but not limited to, differential energy assimilation, expenditure, or allocation. Second, we hypothesized that the timing of reproductive maturity is determined by resource availability, predicting that snakes consuming more food would attain maturity earlier than snakes consuming less food. Finally, we serially measured plasma T levels to determine whether the onset of intersexual differences in plasma T levels are concurrent with an onset in intersexual differences in growth. While not definitive, this resultwould aid in evaluating the possibility that $\mathrm{T}$ is a contributing factor to SSD in C. atrox.

\section{MATERIALS AND METHODS}

\section{Animal collection and care}

In August 2000, we collected 32 neonate $C$. atrox from a $10 \mathrm{~km}$ road in Pinal Co., AZ. These snakes had not yet fed, as meals are visually detectable in snakes of this age ( $<3$ weeks). We also obtained 18 neonates from six pregnant female $C$. atrox collected from an area within $5 \mathrm{~km}$ of the road. We maintained the 50 neonates in a temperature-controlled chamber in individual cages $(54 \mathrm{x}$ $23 \mathrm{~cm}$, Freedom Breeder, Turlock, CA) with subsurface heating at one end and water available ad libitum. When snakes reached ca. $60 \mathrm{~cm}$ snout-vent length (SVL), they were moved to similar but 
larger $(54 \times 40 \mathrm{~cm})$ cages. Snakes experienced the following heat/light schedules: mid-Apr to midOct: ambient temperature $=25 \pm 1^{\circ} \mathrm{C}$, scotophase 700-1900, supplemental heat on constantly; midOct to mid-Dec and mid-Feb to mid-Apr: ambient temperature $=25 \pm 1^{\circ} \mathrm{C}$, scotophase 700-1900, supplemental heat on during scotophase; mid-Dec to mid-Feb: ambient temperature $=16 \pm 1^{\circ} \mathrm{C}$, no scotophase, supplemental heat off (to simulate an overwintering period). Over the course of the 2year experiment (August 2000-July 2002), six snakes either died or consistently refused food, so data from these animals were not included in the analysis. All animal use was approved by the Arizona State University Institutional Animal Care and Use Committee (Protocol 01-617R).

\section{Experimental design}

Snakes were randomly assigned to one of two treatment groups: high intake (14 females and 9 males) and low intake (9 females and 12 males). High-intake snakes received one mouse per week and low-intake snakes received one mouse every 3 weeks. Before feeding, we recorded the mass of each mouse. The size of mice was progressively increased to follow the growth of the snakes until about March 2001; thereafter, mice consistently weighed 25-35 g. All snakes within each treatment group consumed similar total masses of mice over the course of the study. Snakes were not fed during the overwintering periods (mid-Dec to mid-Feb).

In April 2002, coinciding with the natural mating season and when snakes were 1.5 years old, we conducted breeding trials to assess reproductive activity. We placed males in females' cages for 2-day periods, checked the pairs twice daily, and recorded any copulations. Snakes were

randomly rotated such that each male was paired with each female at least twice; however, high-intake males were paired only with high-intake females, and low-intake males were paired only with low-intake females. Following the breeding trials, we assessed female reproductive condition each month with ultrasonography, and measured the masses and snout-vent lengths of all resultant offspring.

\section{Measurements and blood collection}

Every 6 weeks, we collected a blood sample and measured the SVL $( \pm 0.1 \mathrm{~cm})$ and mass $( \pm 0.5$ g) of each snake. Blood collection always occurred between 0800 and 1200 to control for circadian rhythms in hormone levels. Snakes were weighed, coaxed into plastic tubes, and bled from the caudal vein $(0.25-1.0 \mathrm{ml})$ with a heparinized syringe within $5 \mathrm{~min}$ of removal from their cages. Blood was immediately centrifuged, and plasma was collected and stored at $-80^{\circ} \mathrm{C}$ until radioimmunoassay could be performed. We then placed the snakes in a squeeze box (Quinn and Jones, '74), traced a line on the plastic top following the spine from snout to vent, and measured the line with a cloth measuring tape ( $=\mathrm{SVL})$.

\section{Radioimmunoassay}

We assayed blood samples for $\mathrm{T}$ because it is the primary androgen in this species, and other androgens such as dihydrotestosterone (DHT) are found in direct proportion to $T$ but in much smaller quantities (Schuett et al., 2002, in press). We measured plasma levels of $T$ in half the blood samples from males (every other sampling period), and at select sampling periods for females (October 2000, January 2001, October 2001, January 2002). Plasma volumes of $20 \mu \mathrm{l}$ were refrigerated overnight with distilled water and $2000 \mathrm{cpm}$ of ${ }^{3} \mathrm{H}$-testosterone $\left({ }^{3} \mathrm{H}-\mathrm{T}\right.$, NEN Life Science Products Inc., Boston, MA, Catalogue NET553) for individual recovery determination. We utilized an etherethanolhexane extraction protocol in order to remove proteins and lipids that might interfere with antibody-hormone interactions (Taylor et al., 2004). Samples were extracted in $3 \mathrm{ml}$ diethyl ether, the ether fractions were removed and dried with a stream of nitrogen gas in a hot water bath, and the samples were resuspended in $1 \mathrm{ml} 90 \%$ ethanol and refrigerated overnight. Samples were then extracted with $2 \mathrm{ml}$ hexanes, the ethanol fractions were removed and dried with nitrogen gas, and the samples were resuspended in $0.5 \mathrm{ml}$ assay buffer (phosphate-buffered saline with gelatin). We used $200 \mu \mathrm{l}$ in duplicate for the assay and an additional $50 \mu \mathrm{l}$ for individual recoveries. For the assay, we added $100 \mu \mathrm{l}^{3} \mathrm{H}-\mathrm{T}$ and $100 \mu \mathrm{l}$ antibody (Wein Laboratories Inc., Succasauna, NJ, Catalogue T-3003) 
to each duplicate sample, $100 \%$ bounds, and triplicate standard curve, and refrigerated them overnight. This antibody exhibits moderate cross-reactivity with DHT; however, since DHT levels parallel $\mathrm{T}$ cycles and are so low in C. atrox in comparison to $\mathrm{T}$ (Schuett et al., in press), the significance of any cross-reactivity between T and DHT would be minor. We separated bound and unbound $\mathrm{T}$ with dextran-coated charcoal and added the bound fraction to scintillation vials. We added $3 \mathrm{ml}$ scintillation fluid, waited $12 \mathrm{hr}$, and counted the samples in a Beckman scintillation counter. Final steroid levels were calculated from a cubic spline curve fitted to standard curve values, and sample values were adjusted for individual recoveries. Samples from males and females were analyzed in two separate assays; the intra-assay coefficient of variation was $10 \%$, and the interassay coefficient of variation was $0.3 \%$. Mean percent recovery was $60 \%$, and accuracy was $98 \%$. All samples had detectable levels of $\mathrm{T}$.

\section{Data analysis}

Statistical tests were performed using SAS (SAS Institute, Cary, IN, version 8.2), and data were subjected to tests for normality, homogeneity of variances, and presence of outliers prior to inferential tests. SVLs and masses were Intransformed to homogenize variances, but the data in the figures are shown back-transformed to original values.

We analyzed growth (i.e., changes in SVL) using the GLM procedure in SAS to perform a repeated measures analysis of variance (RMANOVA) with sex and diet as between-subjects factors, time as the within-subjects factor, and In-transformed SVL as the dependent variable. For mass gain, we performed a similar analysis, with In-transformed mass as the dependent variable. Mauchly's Criterion for Sphericity was violated for both the SVL and mass analyses; we therefore used multivariate Wilks' Lambda tests rather than univariate tests ( $O$ 'Brian and Kaiser, ' 85$)$. We performed Tukey post-hoc comparisons of SVL and mass between males and females in each treatment group at each time period.

We analyzed T data using the MIXED procedure to perform RMANOVA with sex and diet as

between-subjects factors, time as the within-subjects factor, and T concentration $(\mathrm{ng} / \mathrm{ml})$ as the dependent variable. The fact that we did not measure female $T$ levels at all of the time points that we measured male $T$ levels (see radioimmunoassay section above) resulted in missing data for the females. The MIXED procedure allows analysis of data sets with missing data (Littell et al., '96). PROC MIXED inferences were made using the unstructured covariance structure because this minimized the Akaike's Information and Schwarz' Bayesian Criteria (Littell et al., '96). Post-hoc comparisons of $\mathrm{T}$ levels between males and females and between high- and low-intake males at each time period were made with univariate t-tests adjusted for an experimentwise Type 1 error rate of 0.05 .

\section{RESULTS}

\section{Growth and mass gain}

At the beginning of the experiment, there were no significant differences in SVL between snakes in each treatment group, but they diverged by the second measurement period, with highintake snakes growing faster in SVL than low-intake snakes (Fig. 2). The ANOVA model detected a significant effect of diet on SVL, but sex and the sex $x$ diet interaction were not significant, indicating no overall sex differences in growth (Table 1 ). There were significant effects of time and the time $x$ treatment interaction on snake SVL, indicating that SVL changed over time and that the high- and low-intake treatment groups changed differently over time. There was also a significant time $x$ sex interaction, indicating that the sexes changed differently in SVL over time. These sex differences were manifest only in the low-intake group, where females were slightly shorter than males at the beginning of the experiment because small females were by chance assigned to the low-intake group during random assignment of subjects to groups. Tukey post-hoc tests showed that this sex difference was significant early in the experiment but disappeared over time (Fig. 2). The high-intake snakes showed no sex differences in SVL throughout the experiment. 
Results for the mass gain analysis were similar. By the second measurement period, high-intake snakes were significantly heavier than low-intake snakes (Fig. 3). The ANOVA model detected a significant effect of diet on mass (Table 2). Sex did not significantly affect mass gain, but the sex-diet interaction was significant, indicating that the sexes gained mass differently in each treatment group. In the low-intake group, females weighed less than males at the beginning of the experiment but this difference disappeared over time, while in the high-intake group, females actually became heavier than males toward the end of the experiment (Fig. 3). There were significant effects of time and the time $x$ treatment interaction on snake mass, indicating that mass changed over time and that the high- and low-intake treatment groups changed differently over time. However, the time $x$ sex interaction was not significant, which means that males and females gained mass similarly over time.

\section{Testosterone levels}

Males had higher plasma T levels than females, and high-intake males had higher $\mathrm{T}$ levels than low-intake males at several time periods (Fig. 4). There were significant main effects of time, treatment, and sex, and all interactions were also significant (Table 3). This indicates that T levels changed over time, were different between the treatment groups and between the sexes, and changed differently over time in each treatment group and sex. Post-hoc tests revealed that males and females had similar T levels before 1/01, and males had higher T thereafter (Fig. 4). Post-hoc tests also revealed that males in the two treatment groups had significantly different levels of $T$ at three of eight measurement periods (January 2001, July 2001, January 2002). These differences appear to be the result of higher T peaks in snakes from the high-intake group during these months (Fig. 4). This pattern is similar to the two peaks in $T$ in free-ranging males, except that the peaks in this experiment occurred in January and July, whereas they occur in March and August in the wild (Taylor et al., 2004). The difference in timing of the T peaks most likely reflects differences in environmental cues in the laboratory and field. By July 2001 (age = 1 year), the high-intake males had circulating levels of T similar to those of free-ranging adult males (Taylor et al., 2004). Females consistently had low levels of T, similar to free-ranging females (Taylor et al., 2004).

\section{Sexual maturity}

In the breeding trials, six high-intake females copulated with five high-intake males. In contrast, no low-intake snakes copulated. Of the six high-intake females that copulated, four became pregnant. Their litter sizes were similar to those of wild female $C$. atrox from a study site within $5 \mathrm{~km}$ of the area where snakes in this study were collected (lab: $\mathrm{n}=4$ litters; mean number of neonates $=$ $4.0 \pm 1.8$; field: $n=18$ litters; mean number of neonates $=4.5 \pm 1.6$; Taylor and DeNardo, in press). However, the offspring of the high-intake females were larger than offspring born to wild females (lab: $\mathrm{n}=15$ neonates, mean $\mathrm{SVL}=32.5 \pm 1.2 \mathrm{~cm}$, range $=30.8-34.5 \mathrm{~cm}$; field: $\mathrm{n}=81$ neonates; mean $\mathrm{SVL}=28.6 \mathrm{~cm} \pm 1.5$, range $=19.5-35.0 \mathrm{~cm} ; \mathrm{t}=-5.97, \mathrm{p}<0.0001)$, and were also heavier than wild offspring (lab: mean mass $=30.8 \pm 5.9 \mathrm{~g}$, range $=21.0-44.5 \mathrm{~g}$; field: mean mass $=20.0 \pm 3.6 \mathrm{~g}$, range $=$ 9.0-36.0g; $\mathrm{t}=-7.68, \mathrm{p}<0.0001)$.

\section{DISCUSSION}

In this study, we experimentally examined the growth trajectories of C. atrox, a species of snake with male-biased SSD. High-intake snakes grew and gained mass faster than low-intake snakes, but males did not grow or gain mass more quickly than females. These results support the hypothesis of resource-dependent growth but do not support the hypothesis of sex differences in growth associated with male-biased SSD. High-intake snakes reached reproductive maturity earlier than low-intake snakes, supporting the hypothesis that attainment of maturity is affected by resource availability rather than age. Finally, high-intake males had higher T levels than low-intake males, and all females had low $\mathrm{T}$ throughout the experiment. The presence of a sex difference in $\mathrm{T}$ 
levels combined with a lack of a sex difference in growth suggests that T may not affect growth, and therefore may not be involved in SSD.

The high-intake snakes attained adult-typical SVLs in less than 1 year, whereas the lowintake snakes grew along a trajectory that more closely resembled that of wild $C$. atrox, which typically mature in 3-4 years (Fitch and Pisani, '93; Beaupre et al., '98). The smallest female and male we observed mating at our study site were 70 and $75 \mathrm{~cm}$ in SVL, respectively. The high-intake snakes in this experiment reached these sizes by 10 months of age and continued to grow much larger (Fig. 2), but males did not grow faster or become larger than females throughout the study. The high-intake females in this study averaged $100 \mathrm{~cm} \mathrm{SVL}$ (range $=92.7-106.8 \mathrm{~cm}$ ) at only 2 years of age, a size that greatly exceeds that of wild adult females at our study site $(n=67$; mean $S V L=82.4$ $\mathrm{cm}$; range $=70-94 \mathrm{~cm}$ ). In fact, the size of these high-intake females even exceeds that of adult males from the same site $(n=104 ;$ mean $S V L=95.3 \mathrm{~cm}$; range $=75-131 \mathrm{~cm} ; E$. Taylor and $D$. DeNardo, unpublished data).

The results for mass were similar: high-intake snakes gained mass more quickly than lowintake snakes, but males did not gain mass faster than females. In fact, from October 2001 until the end of the study, the high-intake females actually weighed more than the high-intake males, a trend that conflicts with patterns observed in the wild. This mass increase was not the result of deposition of yolk into eggs in preparation for reproduction, as ultrasonography showed that reproductive females did not initiate vitellogenesis until April 2002. The mass difference may be the result of differential retention of water, differential assimilation of food, or some other unknown factor. At only 2 years of age, the mean mass of the high-intake females was 892g (range= 747-988g) far higher than that of wild females ( $n=67$; mean mass $=360 \mathrm{~g}$; range $=195-510 \mathrm{~g}$ ) and even wild males $(n=104$; mean mass $=533 \mathrm{~g}$; range $=220-1192 \mathrm{~g}$; E. Taylor and D. DeNardo, unpublished data $)$ at our study site. The lack of sex differences in growth and mass gain in snakes fed the same diet and housed under the same conditions suggests that the SSD observed in wild snakes is a plastic rather than fixed phenomenon.

Androgens (e.g., T) are involved in the expression of many sexually dimorphic characters, such as comb development in chickens (Rath et al., '96), dewlap and ventral patch coloration in tree lizards (Hews and Moore, '95), and sexual dimorphism of the vertebrate brain (Gorski et al., '78; Breedlove, '92). Testosterone generally contributes to the larger body size and muscle mass of males relative to females (Bardin and Catterall, '81; Joubert et al., '94). Since rattlesnakes exhibit male-biased SSD (Klauber, '72), it is possible that T may promote growth in the male C. atrox. If $T$ is the physiological factor responsible for SSD, then increases in T associated with sexual maturity in males should lead to increased growth of males relative to females thereafter (Fig. 1). However, our findings are not consistent with this prediction in that, at maturity, males consistently had higher plasma T levels (typical of free-ranging adult males; Taylor et al., 2004) but never showed higher growth rates than females (Figs. 2-4). While we merely demonstrate a lack of correlation between intersexual differences in $T$ levels and growth, these results suggest that $\mathrm{T}$ is not directly responsible for growth. It is possible that T indirectly affects SSD, for example by stimulating increased activity of males, which then could lead to increased foraging success and/or muscle mass, an effect which would not occur under laboratory conditions. However, activity also entails increased energy expenditure and therefore the dramatic difference in size between the male and female $C$. atrox is unlikely to be the result of differences in activity alone. Possible direct and indirect roles of $T$ in growth must be further tested by experimentally manipulating $T$ levels in both the laboratoryhoused and free-ranging maleC. atrox.

The fact that high-intake males had higher Tlevels than low-intake males likely reflects the fact that they attained reproductive maturity earlier. At the time of the breeding trials (April 2002), low-intake snakes were smaller in size than snakes we typically observe copulating in the wild, 
whereas high-intake snakes were as large as the largestsnakes we find in the wild. This supports the idea that there is a minimum size rather than a minimum age necessary for attainment of sexual maturity, an idea that has been supported in other studies on ectotherms. For example, Ford and Seigel ('94) found that female snakes of the genusElaphe matured early when raised on a high-intake diet. The mechanism by which attainment of a certain body size permits sexual maturity is unknown. One possibility is that attainment of acertain SVL is correlated with accumulation of acritical amount of fat reserves. In mammals, the hormone leptin is secreted from adipose cells in proportion to the amount of fat present and signals the central nervous system to begin production of gonadotropins at the time of puberty (Yu et al., '97), which then initiate the cascade of reproductive events. Indeed, prepubertal female mice injected with leptin attain reproductive maturity at an earlier age and smaller size (Chehab et al., '97). The effects of leptin on reproduction or attainment of maturity in reptilesare unknown.

There are several problems with utilizing copulation and pregnancy as indices for sexual maturation. First, reproductive behavior is seasonal in C. atrox, and therefore snakes may have been physiologically capable of reproduction much sooner than we detected if the appropriate environmental stimulus (warming after a cooloverwintering period) had been provided earlier. Thus, reproductive activity during this experiment confirms sexual maturity but does notnecessarily reflect the onset of sexual maturity, which might be better estimated by size relative to known reproducing animals in the wild. It ispossible that the low-intake snakes were capable of producing viable gametes but did not copulatefor another, unknown reason or that one sex in the low-intake group was mature but the otherwas not. However, combining our data for reproduction, male testosterone levels, and growth relative to free-ranging wild snakes provides convincing evidence that snakes that consume more food reach sexual maturity earlier. Further tests are necessary to more fully understand theonset of sexual maturity and the mechanisms thatregulate it.

Our results show that $C$. atrox, like many otherspecies of snake, exhibit resource-dependent growth. In turn, attainment of reproductive maturity depends on this resource-dependent growth. The high-intake snakes surpassed the average SVL and mass of wild snakes and became reproductively mature during the study, yet SSD never developed. In fact, our results show that female $C$. atrox are capable of attaining male-like body sizes under laboratory conditions, indicating that the SSD present in wild $C$. atrox (outlined in Fig. 1) may be the result of intersexual differencesin energy intake and/or expenditure. The most likely factor that could contribute to the small size of females in the wild is the high cost of reproduction relative to males (Beaupre, 2002). Beaupre and Duvall ('98) showed that the reproductive female $C$. atrox have higher energy requirements than non-reproductive females (an average non-reproductive female snake requires $4593 \mathrm{~J} /$ day, while a reproductive snake requires $6580 \mathrm{~J} /$ day). Following parturition, females are emaciated and allocate acquired energy toward replenishing that lost during reproduction, and little growthoccurs (Beaupre, 2002), resulting in slowed growth relative to males after maturity (Fig. 1). Thus, SSD in rattlesnakes may be the consequence of differential energy expenditure between the sexes, and may not be the result of sexual selectionfavoring growth in males. In our study, reproductive investment by some of the high-intake females did not lead to SSD, probably reflecting the extremely high energy intake of these animals relative to the resource-limited condition in nature. Further research regarding the mechanisms that control the allocation of energy into storage versus growth and the roles that such mechanisms play in SSD is needed. In addition, future research efforts should explore other possible factors that might contribute to the small size of females relative to males in natural populations, such as potential differences in energyacquisition, thermoregulation, or activity levels.

\section{ACKNOWLEDGMENTS}


We thank C. Ellermeier and D. Gaillard forassistance in conducting the breeding trials. M. Feldner provided valuable assistance in bleeding and measuring snakes. We thank G. Schuettfor experimental design advice, D. Jenningsfor radioimmunoassay (RIA) advice, and M. Mooreand M. Orchinik for use of RIA facilities. Comments from S. Beaupre, O. Lourdais, andmembers of the DeNardo laboratory improved themanuscript. LITERATURE CITED

Andersson M. 1994. Sexual selection. rinceton, NJ: Princeton University Press.

Andrews RM. 1982. Patterns of growth in reptiles. In: Gans C, Pough FH, editors. Biology of the Reptilia, Vol. 13. New York: Academic Press. p 273-334.

Bardin CW, Catterall JF. 1981. Testosterone: a major determinant of extragenital sexual dimorphism. Science 211:12851294.

Beaupre SJ. 2002. Modeling time-energy allocation in vipers: individual responses to environmental variation and implications for populations. In: Schuett GW, Hoggren M, Douglas ME, Greene HW, editors. Biology of the vipers. Eagle Mountain, UT: Eagle Mountain Publishing.p 463-481.

Beaupre SJ, Duvall D. 1998. Variation in oxygen consumption of the western diamondback rattlesnake (Crotalus atrox): implications for sexual size dimorphism. J Comp Physiol B168:497-506.

Beaupre SJ, Duvall D, O’Leile J. 1998. Ontogenetic variation in growth and sexual size dimorphism in a central Arizona population of the western diamondback rattlesnake (Crotalus atrox). Copeia 1998:40-47.

Bonnet X, Shine R, Naulleau G, Thiburce C. 2001. Plastic vipers: influence of food intake on the size and shape of Gaboon vipers (Bitis gabonica). J Zool 255:341-351.

Breedlove SM. 1992. Sexual dimorphism in the vertebratebrain. J Neurosci 12:4133-4142.

Chehab FF, Mounzih K, Lu RH, Lim ME. 1997. Early onset of reproductive function in normal female mice treated with leptin. Science 275:88-90.

Crews D, Diamond MA, Whittier J, Mason R. 1985. Small malebody size in garter snakes depends on testes. Am J Physiol 249:R62-R66.

Darwin C. 1871. The descent of man and selection in relationto sex. London: John Murray.

Duvall D, Beaupre SJ. 1998. Sexual strategy and size dimorphism in rattlesnakes: integrating proximate and ultimate causation. Am Zool 38:151-164.

Fitch HS. 1981. Sexual size differences in reptiles. Univ Kans Mus Nat Hist Misc Publ 70:1-71.

Fitch HS, Pisani GR. 1993. Life history traits of the Western Diamondback Rattlesnake (Crotalus atrox) studied from roundup samples in Oklahoma. Occ Pap Kans Mus Nat Hist 156:1-24.

Ford NB, Seigel RA. 1989. Phenotypic plasticity in reproductive traits: evidence from a viviparous snake. Ecology 70:17681774.

Ford NB, Seigel RA. 1994. An experimental study of the tradeoffs between age and size at maturity: effects of energy availability. Funct Ecol 8:91-96.

Forsman A, Lindell LE. 1996. Resource dependent growth and body condition dynamics in juvenile snakes: an experiment. Oecologia 108:669-675.

Gorski RA, Gordon JH, Shryne JE, Southam AM. 1978. Evidence for a morphological sex difference within the medial preoptic area of the rat brain. Brain Res 148:333-346.

Hews DK, Moore MC. 1995. Influence of androgens on differentiation of secondary sex characters in tree lizards, Urosaurus ornatus. Gen Comp Endocrinol 97:86-102.

Joubert Y, Tobin C, Lebart MC. 1994. Testosterone-induced masculinization of the rat levator ani muscle during puberty. Dev Biol 162:104-110.

Klauber LM. 1972. Rattlesnakes: their habits, life histories, and influence on mankind. Berkeley, CA: University of California Press.

Lerner DT, Mason RT. 2001. The influence of sex steroids on the sexual size dimorphism in the Red-Spotted Garter Snake, Thamnophis sirtalis concinnus. Gen Comp Endocrinol 124:218-225.

Littell RC, Milliken GA, Stroup WW, Wolfinger RD. 1996. SAS system for mixed models. Cary, NC: SAS Institute, Inc.

Lourdais O, Bonnet X, Shine R, DeNardo D, Naulleau G, Guillon M. 2002. Capital-breeding and reproductive effort in a variable environment: a longitudinal study of a viviparous snake. J Anim Ecol 71:470-479.

Madsen T, Shine R. 1994. Costs of reproduction influence the evolution of sexual size dimorphism in snakes. Evolution 48:1389-1397.

O’Brian RG, Kaiser MK. 1985. MANOVA method for analyzing repeated measures designs: an extensive primer. Psychol Bull 97:316-333.

Partridge L, Harvey PH. 1988. The ecological context of lifehistory evolution. Science 241:1449-1455.

Quinn H, Jones JP. 1974. Squeeze box technique formeasuring snakes. Herpetol Rev 5:35.

Rath NC, Huff WE, Balog JM, Bayyari GR. 1996. Effect of gonadal steroids on bone and other physiological parameters of male broiler chickens. Poultry Sci 75:556-562.

Ricklefs RE, Wikelski M. 2002. The physiology/life history nexus. Trends Ecol Evol 17:462-468. 
Schuett GW. 1997. Body size and agonistic experience affect dominance and mating success in male copperheads. Anim Behav 54:213-224.

Schuett GW, Carlisle SL, Holycross AT, Hardy Sr., DL, Van Kirk EA, Murdoch WJ. 2002. Mating system of male Mojave Rattlesnakes (Crotalus scutulatus): seasonal timing of mating, agonistic behavior, spermatogenesis, sexual segment of the kidney, and plasma sex steroids. In: Schuett GW, Hoggren M, Douglas ME, Greene HW, editors. Biology of the vipers. Eagle Mountain, UT: Eagle Mountain Publishing. p 515-532.

Schuett GW, Hardy Sr., DL, Greene HW, Earley RL, Grober MS, Van Kirk EA, Murdoch WJ. In press. Sympatric rattlesnakes with contrasting mating systems show differences in seasonal patterns of plasma sex steroids. Anim Behav.

Scudder-Davis RM, Burghardt GM. 1996. Ontogenetic changes in growth efficiency in laboratory-reared water snakes of the genus Nerodia. The Snake 27:75-84.

Shine R. 1978. Sexual size dimorphism and male combat insnakes. Oecologia 33:269-277. 


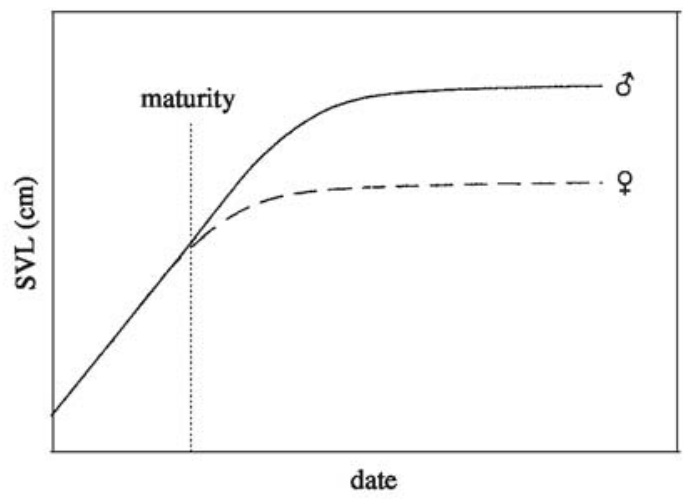

Fig. 1. Schematic of the development of sexual size dimorphism in Crotalus atrox. Juvenile snakes grow at the same rate until sexual maturity, after which males (solid line) grow faster than females (dashed line). The divergence in growth is caused by an unknown factor.

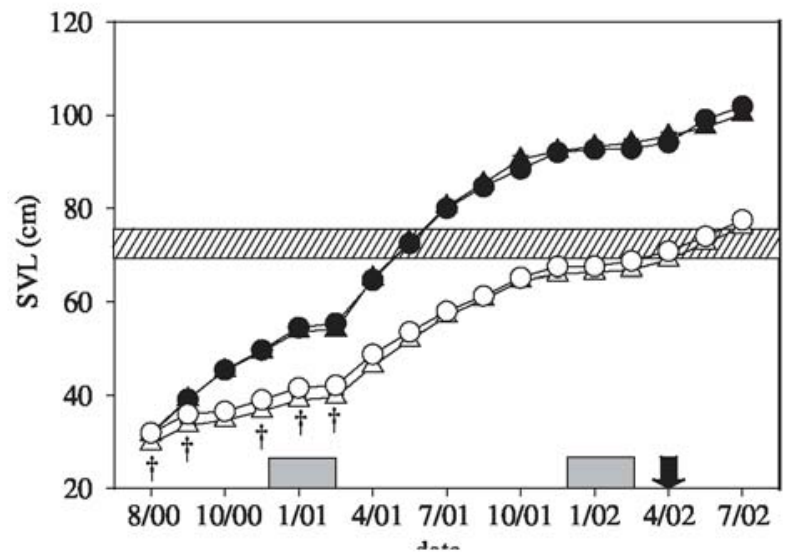

Fig. 2. Growth in snout-vent length (SVL) of Crotalus atrox. High-intake males are black circles and solid lines; high-intake females are black triangles and solid lines; low-intake males are white circles and dashed lines; low-intake females are white triangles and dashed lines. Significant sex differences in the low-intake group are marked by t. The shadowed bars correspond to overwintering periods during which snakes were not fed, the hatched bar denotes the approximate size at which free-ranging snakes attain sexual maturity, and the black arrow denotes the timing of the breeding trials. Values are shown as mean+1 SEM, although error bars are often invisible due to lack of variability among experimental units. 


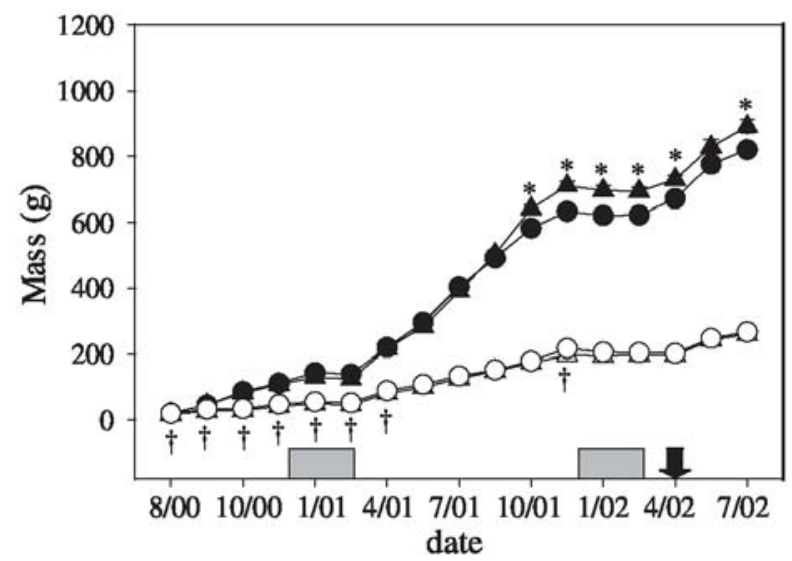

Fig. 3. Change in mass of Crotalus atrox. High-intake males are black circles and solid lines; highintake females are black triangles and solid lines; low-intake males are white circles and dashed lines; low-intake females are white triangles and dashed lines. Significant sex differences in the lowintake group are marked by $t$, in the high-intake group by *. The shadowed bars correspond to overwintering periods during which snakes were not fed, and the black arrow denotes the timing of the breeding trials. Values are shown as mean+1 SEM, although error bars are often invisible due to lack of variability among experimental units.

TABLE 1. Repeated-measures ANOVA table for effects of diet, sex, time, and their interactions on In-transformed SVL (cm) in Crotalus atrox

\begin{tabular}{|c|c|c|c|c|}
\hline Between subjects & $\mathrm{df}$ & Type III S.S. & $F$-ratio & $P$-value \\
\hline Diet & 1 & 13.900 & 577.41 & $<0.0001$ \\
\hline Sex & 1 & 0.069 & 2.86 & 0.100 \\
\hline Diet $\times$ sex & 1 & 0.062 & 2.59 & 0.116 \\
\hline Error & 40 & 0.963 & & \\
\hline Within subjects & df & Wilks' $\lambda$ & $F$-ratio & $P$-value \\
\hline Time & 16.25 & 0.002 & 773.2 & $<0.0001$ \\
\hline Time $\times$ diet & 16.25 & 0.061 & 23.90 & $<0.0001$ \\
\hline Time $\times$ sex & 16.25 & 0.366 & 2.70 & 0.013 \\
\hline Time $\times$ sex $\times$ diet & 16.25 & 0.474 & 1.73 & 0.106 \\
\hline
\end{tabular}

TABLE 2. Repeated-measures ANOVA table for effects of diet, sex, time, and their interactions on ln-transformed mass $(\mathrm{g})$ in Crotalus atrox

\begin{tabular}{lcccc}
\hline Between subjects & df & Type III S.S. & $F$-ratio & $P$-value \\
\hline Diet & 1 & 180.247 & 2627.17 & $<.84$ \\
Sex & 1 & 0.264 & 6.00 & 0.060 \\
Diet $\times$ sex & 1 & 0.411 & & 0.019 \\
Error & 40 & 2.744 & $F$-ratio & $P$-value \\
Within subjects & df & Wilks' $\lambda$ & 965.42 & $<0.0001$ \\
Time & 16.25 & 0.002 & 59.02 & $<0.0001$ \\
Time $\times$ diet & 16.25 & 0.026 & 1.34 & 0.248 \\
Time $\times$ sex & 16.25 & 0.538 & 2.31 & 0.030 \\
Time $\times$ sex $\times$ diet & 16.25 & 0.404 & & \\
\hline
\end{tabular}




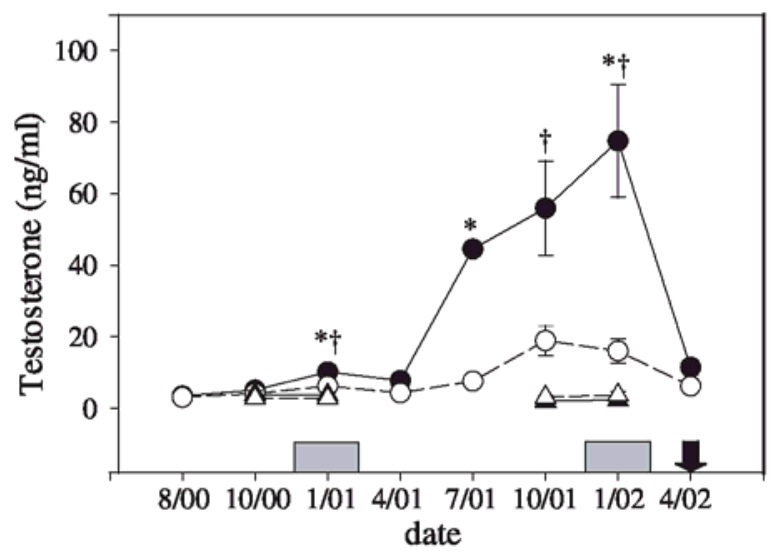

Fig. 4. Testosterone levels of male and female snakes in each treatment group. High-intake males are black circles and solid lines; high-intake females are black triangles and solid lines; low-intake males are white circles and dashed lines; low-intake females are white triangles and dashed lines. Significant differences between males and females are marked by t; those between high- and lowintake males by *. The shadowed bars correspond to overwintering periods, and the black arrow denotes the timing of the breeding trials. Values are shown as mean +1 SEM. Plasma T was only measured at four time periods in samples from females.

TABLE 3. Repeated-measures ANOVA table for effects of diet, sex, time, and their interactions on plasma testosterone levels (ng/ml) in Crotalus atrox

\begin{tabular}{lccc}
\hline Between subjects & df & $F$-ratio & $P$-value \\
\hline Diet & 1.25 & 5.15 & 0.032 \\
Sex & 1.25 & 18.86 & $<0.001$ \\
Diet $\times$ sex & 1.25 & 6.91 & 0.014 \\
Within subjects & df & $F$-ratio & $P$-value \\
\hline Time & 7.25 & 75.93 & $<0.0001$ \\
Time $\times$ diet & 7.25 & 46.28 & $<0.0001$ \\
Time $\times$ sex & 3.25 & 6.75 & 0.002 \\
Time $\times$ sex $\times$ diet & 3.25 & 3.36 & 0.035 \\
\hline
\end{tabular}

